\title{
The Continuum Stored Energy for Constitutive Modeling Finite Deformations of Polymeric Materials
}

\author{
Fuzhang Zhao \\ APD Optima Study, Lake Forest, CA, USA \\ Email: fuzhangzhao@yahoo.com
}

How to cite this paper: Zhao, F.Z. (2017) The Continuum Stored Energy for Constitutive Modeling Finite Deformations of Polymeric Materials. Advances in Pure Mathematics, 7, 597-613.

https://doi.org/10.4236/apm.2017.710036

Received: October 4, 2017

Accepted: October 28, 2017

Published: October 31, 2017

Copyright $\odot 2017$ by author and Scientific Research Publishing Inc. This work is licensed under the Creative Commons Attribution International License (CC BY 4.0).

http://creativecommons.org/licenses/by/4.0/

\section{(c) (i) Open Access}

\begin{abstract}
With symmetries measured by the Lie group and curvatures revealed by differential geometry, the continuum stored energy function possesses a translational deformation component, a rotational deformation component, and an ellipsoidal volumetric deformation component. The function, originally developed for elastomeric polymers, has been extended to model brittle and ductile polymers. The function fits uniaxial tension testing data for brittle, ductile, and elastomeric polymers, and elucidates deformation mechanisms. A clear distinction in damage modes between brittle and ductile deformations has been captured. The von Mises equivalent stress has been evaluated by the function and the newly discovered break-even stretch. Common practices of constitutive modeling, relevant features of existing models and testing methods, and a new perspective on the finite elasticity-plasticity theory have also been offered.
\end{abstract}

\section{Keywords}

Break-Even Stretch, Continuum Stored Energy, Damage Mode, Deformation Mechanism, Finite Elasticity-Plasticity Theory, Polymeric Material

\section{Introduction}

Constitutive modeling for finite deformations of polymeric materials requires accurate theoretical predictions combined with experimental characterizations. Experimental tests cannot characterize materials in all deformation modes although experimental tests are more complete than theoretical models. As a result, a theoretical model is fitted with experimental data tested in certain deformation modes and the fitted model predicts deformations in untested 
modes. Thus, the theoretical development of constitutive models and their numerical implementations into finite element methods are crucial in designs and analyses of polymer components, which have been heavily applied in the aerospace, automotive, consumer electronics, medical device, and space exploration industries.

For elastic-plastic constitutive models, great progress has been made in continuum mechanics. Earlier achievements in kinematics, conservation principles, and constitutive relations were archived in the classic treatise on continuum mechanics by Truesdell and Noll (1965) [1]. Fundamental theories of plasticity were presented in the monographs by Hill (1950) [2], Simo and Hughes (1998) [3], and Haupt (2002) [4].

Incremental strain as well as total strain theories of plasticity, featuring assumptions, models, and computer implementations, have been comprehensively reviewed by Armen (1979) [5]. Rate-independent theories of plasticity, emphasizing the strain-based approach over the stress-based approach, have been critically reviewed by Naghdi (1990) [6]. Both hyperelastic-based and hypoelastic-based theories of plasticity, highlighting theoretical inconsistencies and physical pertinent formulations, have been concisely reviewed by Xiao, Bruhns, and Meyers (2006) [7]. Constitutive theories of plasticity and viscoplasticity, with thermodynamic and alternative frameworks, have been briefly reviewed by Chaboche (2008) [8]. Recent phenomenological and physical theories of plasticity and viscoplasticity for polymers, including volumetric damage, have been thoroughly summarized by Ponçot, Addiego, and Dahoun (2013) [9].

Accurate constitutive relations and further computer implementations are crucial to maintaining the required numerical properties of accuracy, efficiency, and stability. Among them, accuracy is one of the most important numerical properties. The five key elements of existing elastic-plastic constitutive relations are stress-strain relations, decompositions of strains, yield criteria, hardening laws, and flow rules. The current theories of plasticity can only maintain the numerical stability in terms of normality of the incremental plastic strain vector and convexity of initial and subsequent yield surfaces but cannot accurately predict plastic deformations in general modes, as noticed by Michno and Findley (1976) [10]. Polymers under loadings are primarily involved with chain stretching, chain rotating, and volumetric strengthening or damaging and the physical relevant behaviors of polymers need to be captured. Some semicrystalline polymers can sustain $3000 \%$ to $4000 \%$ of stretches without breaking, which is far beyond the deformation range for regular metallic materials (except for super-plastic metals) and a truly finite deformation theory of plasticity is needed. For resolving the existing issues of accurate predictions, physical relevancies, and finite elastic-plastic deformations, a physically relevant and mathematically covariant stored energy function is needed.

The continuum stored energy (CSE) function, originally developed for modeling elastomeric polymers by Zhao (2016) [11], has been extended to 
model brittle and ductile polymers. The main objectives, therefore, are to fit the CSE function to experimental data for three types of isotropic polymer materials, to study their deformation mechanisms and damage modes, to examine the validity of von Mises equivalent stress and equivalent strain equations, and to recommend a new approach for the finite elasticity-plasticity theory.

\section{Continuum Stored Energy for Isotropic Polymers}

For isothermal processes, the general CSE function for isotropic materials at finite deformations, $\Psi$, has been postulated to be balanced with its stress work done as ${ }^{1}$

$$
\Psi=S: \frac{C}{2}
$$

where the second Piola-Kirchhoff stress tensor, $\boldsymbol{S}$, reads

$$
\boldsymbol{S}=2\left[\left(\frac{\partial \Psi}{\partial I_{1}}+I_{1} \frac{\partial \Psi}{\partial I_{2}}\right) \boldsymbol{I}-\frac{\partial \Psi}{\partial I_{2}} \boldsymbol{C}+I_{3} \frac{\partial \Psi}{\partial I_{3}} \boldsymbol{C}^{-1}\right]
$$

the right Cauchy-Green tensor, $\boldsymbol{C}$, is given by

$$
\boldsymbol{C}=\boldsymbol{F}^{\mathrm{T}} \boldsymbol{F}
$$

and $\boldsymbol{F}$ is the deformation gradient tensor.

The three invariants of right Cauchy-Green tensor, $I_{1}, I_{2}$, and $I_{3}$, are related by

$$
\begin{gathered}
I_{1}=\operatorname{tr} C=\lambda_{1}^{2}+\lambda_{2}^{2}+\lambda_{3}^{2} \\
I_{2}=\frac{1}{2}\left[(\operatorname{tr} C)^{2}-\operatorname{tr} C^{2}\right]=I_{3} \operatorname{tr} C^{-1}=\lambda_{1}^{2} \lambda_{2}^{2}+\lambda_{2}^{2} \lambda_{3}^{2}+\lambda_{3}^{2} \lambda_{1}^{2} \\
I_{3}=\operatorname{det} C=\lambda_{1}^{2} \lambda_{2}^{2} \lambda_{3}^{2}
\end{gathered}
$$

the trace of the tensor $C$ is denoted by $\operatorname{tr} C$, the determinant of the tensor $C$ is denoted by $\operatorname{det} C$, and $\lambda_{1}, \lambda_{2}$, and $\lambda_{3}$ are principal stretches in three mutually orthogonal directions. The stretch vector $\lambda_{a_{0}}$ in the direction of the unit vector $\boldsymbol{a}_{0}$ is defined as

$$
\lambda_{a_{0}}=\boldsymbol{F} \boldsymbol{a}_{0}
$$

with the length $\lambda=\left|\lambda_{a_{0}}\right|$, which has been documented by Holzapfel (2000) [12].

Substituting (2) into (1), simplifying, and rearranging yields the following partial differential equation

$$
\Psi=I_{1} \frac{\partial \Psi}{\partial I_{1}}+2 I_{2} \frac{\partial \Psi}{\partial I_{2}}+3 I_{3} \frac{\partial \Psi}{\partial I_{3}}
$$

Based on Lie group methods, the characteristic system for the partial differential Equation (8) is

$$
\frac{\mathrm{d} I_{1}}{I_{1}}=\frac{\mathrm{d} I_{2}}{2 I_{2}}=\frac{\mathrm{d} I_{3}}{3 I_{3}}=\frac{\mathrm{d} \Psi}{\Psi}
$$

${ }^{1}$ The general CSE function, $\Psi$, is covariant to $\Psi_{E}=\boldsymbol{S}: \boldsymbol{E}$ under the transformation of $\boldsymbol{E}=(\boldsymbol{C}-\boldsymbol{I}) / 2$, which has been emphasized by Zhao (2016) [11]. 
and taking its three first-integrals, $\psi_{1}=I_{2} / I_{1}^{2}, \quad \psi_{2}=I_{3} / I_{1}^{3}$, and $\psi_{3}=\Psi / I_{1}$, the general solution has been obtained and written as

$$
\Psi=I_{1}\left[f\left(I_{2} / I_{1}^{2}\right)+g\left(I_{3} / I_{1}^{3}\right)\right]+c_{0}
$$

where $f$ and $g$ are two arbitrary functions, $c_{0}$ is an integration constant, and the general solution defines a group of CSE functions.

The general solution (10) has two arbitrary functions to be determined for practical applications. Translational, rotational, and ellipsoidal deformations are indispensable components for isotropic polymeric materials. Thus, the curvatures of the three types of deformations have been used to select the two arbitrary functions. By doing so, the particular CSE function turns out to be

$$
\Psi_{p}\left(I_{1}, I_{2}, I_{3}\right)=c_{0}+c_{1} I_{1}+c_{2} \sqrt{I_{2}}+c_{3} \frac{I_{1}^{4}}{I_{3}}
$$

where the three coefficients, $c_{1}, c_{2}$, and $c_{3}$, are unknown constitutive constants to be determined by experimental tests.

For the initially undeformed referential configuration, we have the normalized stretches of $\lambda_{1}=\lambda_{2}=\lambda_{3}=1$, resulting in the three constant invariants of $I_{1}=I_{2}=3$ and $I_{3}=1$ from (4), (5), and (6). The CSE function at the undeformed mode is usually assumed to be zero

$$
\Psi_{p}(3,3,1)=0
$$

Substituting the $c_{0}$ determined by (12) into (11) and collecting terms yields the following three-component CSE function

$$
\Psi_{p}\left(I_{1}, I_{2}, I_{3}\right)=c_{1}\left(I_{1}-3\right)+c_{2}\left(\sqrt{I_{2}}-\sqrt{3}\right)+c_{3}\left(\frac{I_{1}^{4}}{I_{3}}-81\right)
$$

For curve fittings with the CSE function (11), the engineering stress and the true stress as a function of principal stretches in uniaxial tension have been derived, respectively. With the constraint of incompressibility, the engineering stress in uniaxial tension tests, $P_{u}$, as a function of principal stretches reads

$$
P_{u}=\left[2 c_{1}+\frac{1}{\sqrt{2 \lambda^{3}+1}} c_{2}+8\left(\lambda^{2}+2 \lambda^{-1}\right)^{3} c_{3}\right]\left(\lambda-\lambda^{-2}\right)
$$

and the true stress in uniaxial tension tests, $\sigma_{u}=P_{u} \lambda$, as a function of principal stretches is

$$
\sigma_{u}=\left[2 c_{1}+\frac{1}{\sqrt{2 \lambda^{3}+1}} c_{2}+8\left(\lambda^{2}+2 \lambda^{-1}\right)^{3} c_{3}\right]\left(\lambda^{2}-\lambda^{-1}\right)
$$

The true stress in equibiaxial tension tests, $\sigma_{b}$, as a function of principal stretches is

$$
\sigma_{b}=\left[2 c_{1}+\frac{1}{\sqrt{1+2 \lambda^{-6}}} c_{2}+8\left(2 \lambda^{2}+\lambda^{-4}\right)^{3} c_{3}\right]\left(\lambda^{2}-\lambda^{-4}\right)
$$

The true stress in pure shear tests, $\sigma_{s}$, as a function of principal stretches is 


$$
\sigma_{s}=\left[2 c_{1}+\frac{1}{\sqrt{\lambda^{2}+\lambda^{-2}+1}} c_{2}+8\left(\lambda^{2}+\lambda^{-2}+1\right)^{3} c_{3}\right]\left(\lambda^{2}-\lambda^{-2}\right)
$$

For finite elastic-plastic deformations of semi-crystalline polymers, there exists a break-even stretch, $\lambda_{b}$, between translational and rotational deformation curves in uniaxial tension tests. Equating the first term and the second term from either (14) or (15) and solving gives

$$
\lambda_{b}=\frac{1}{2}\left[\left(\frac{c_{2}}{c_{1}}\right)^{2}-4\right]^{\frac{1}{3}}
$$

The break-even stretch will be used to study the von Mises equivalent stress.

\section{Fitting the CSE Function to Testing Data}

The deformation mechanisms in polymers are usually classified as brittle, ductile (necked and unnecked), and elastomeric, as documented by Meyers and Chawla (2009) [13]. The typical load-extension curves for polymeric materials are schematically shown in Figure 1.

\subsection{Modeling for Brittle Polymeric Materials}

General-purpose polystyrene (GPPS), as a brittle polymeric material, deforms mainly elastic and breaks at about $2 \%$ strain, which can be found in the book by Smith (1993) [14]. The uniaxial tension experimental data of GPPS has been used to fit the uniaxial tension model (14) by the linear least square method and the three coefficients have been solved as $c_{1}=362341.54826 \mathrm{MPa}$, $c_{2}=-41595.99417 \mathrm{MPa}$, and $c_{3}=-3237.57424 \mathrm{MPa}$. The comparison between the continuum model and the uniaxial tension test data of GPPS is shown in Figure 2. The CSE function is excellently suited to fit the brittle polymer GPPS.

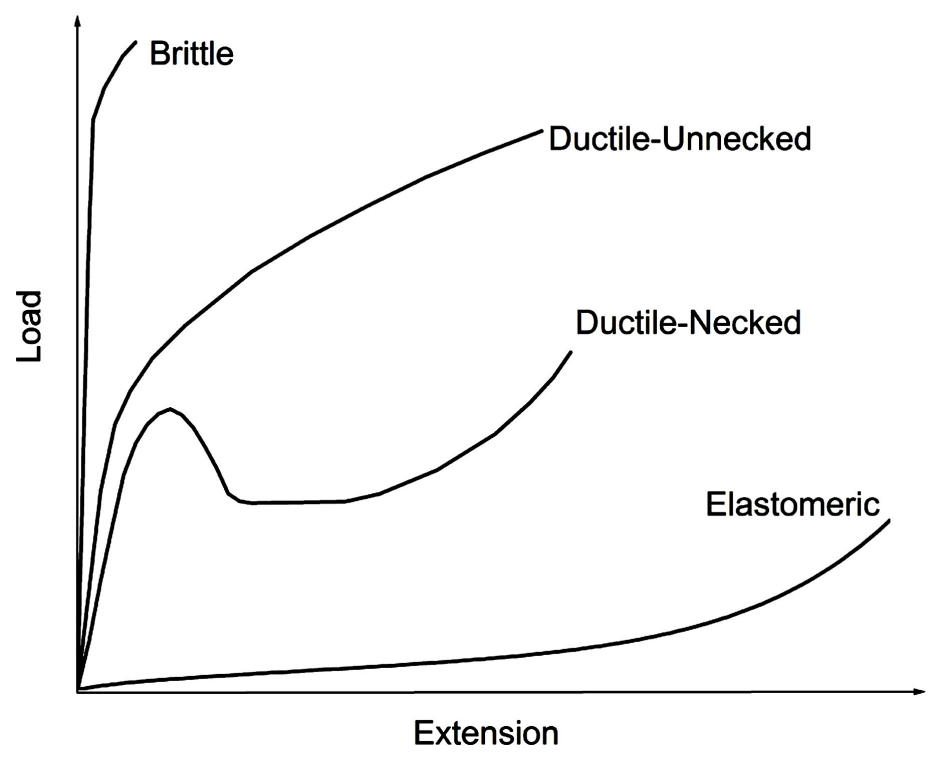

Figure 1. Typical load-extension curves for polymers. 


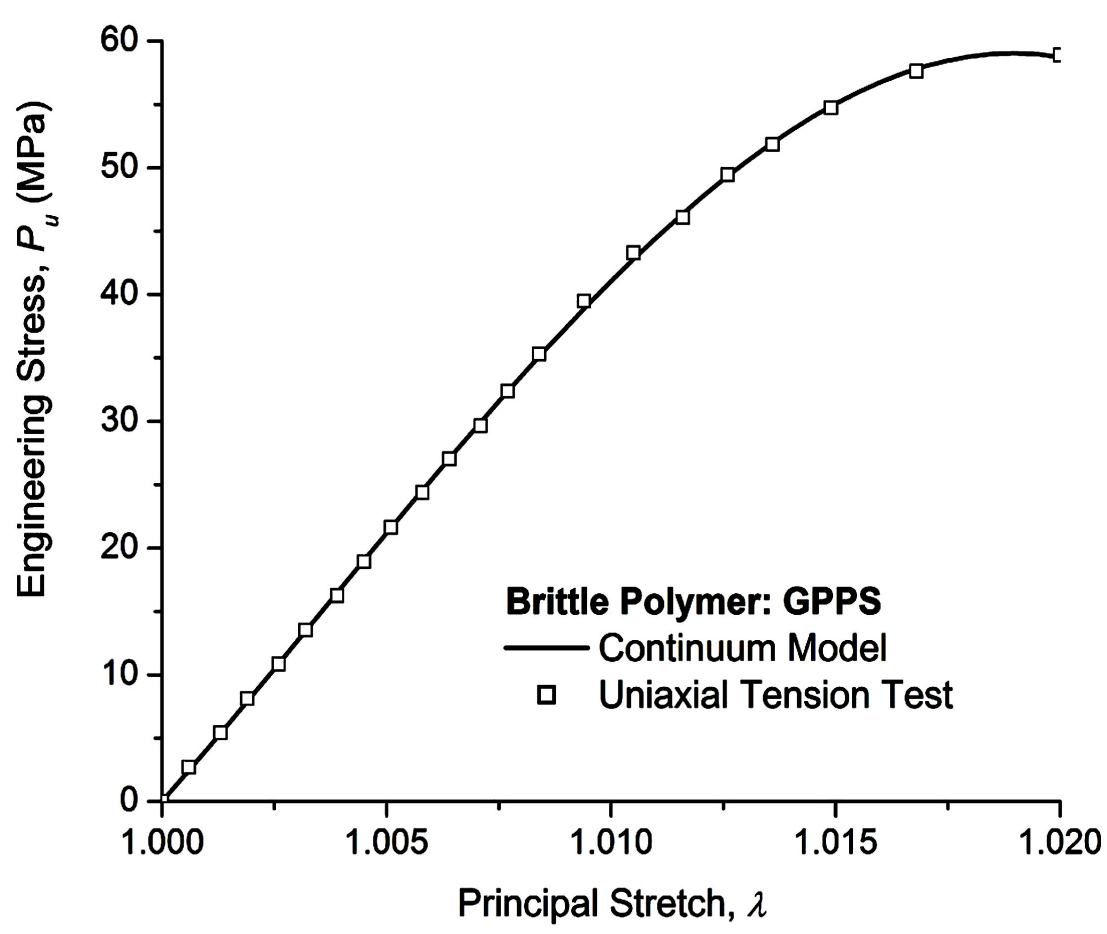

Figure 2. Continuum modeling for brittle polymer material GPPS.

\subsection{Modeling for Ductile Polymeric Materials}

The two ductile polymeric materials selected for uniaxial tension tests are low-density polyethylene (LDPE) and isotactic polypropylene (iPP). Both LDPE and iPP are semicrystalline polymers, containing both amorphous and crystalline phases. Under uniaxial tensions with constant elongation rates and temperatures, semicrystalline polymers are characterized as elastic-plastic materials. The true stress Equation (15) for uniaxial tension tests will be used to model ductile polymeric materials.

The uniaxial tension test of LDPE conducted at the elongation speed of $15 \mathrm{~mm} /$ min by Nitta and Yamana (2012) [15] is selected to fit the continuum constitutive model. The three coefficients, $c_{1}=0.3235954 \mathrm{MPa}, c_{2}=14.63827 \mathrm{MPa}$, and $c_{3}=-1.40847 \times 10^{-10} \mathrm{MPa}$, are obtained. The comparison between the continuum model and the uniaxial tension test data of LDPE is shown in Figure 3. The continuum constitutive model is excellently suited to fit the ductile polymer LDPE.

The uniaxial tension test of iPP conducted at the elongation speed of $5 \mathrm{~mm} /$ min by Nitta and Yamana (2012) [15] is selected to fit the continuum constitutive model. The data for iPP was recorded and converted from both the unnecked and the necked stages during uniaxial tension tests. The three coefficients, $c_{1}=0.1680651 \mathrm{MPa}, c_{2}=60.2789629 \mathrm{MPa}$, and $c_{3}=-1.09346 \times 10^{-12} \mathrm{MPa}$, are obtained. The comparison between the continuum model and the uniaxial tension test data of iPP is shown in Figure 4. The continuum constitutive model is excellently suited to fit the necked ductile polymer iPP. 


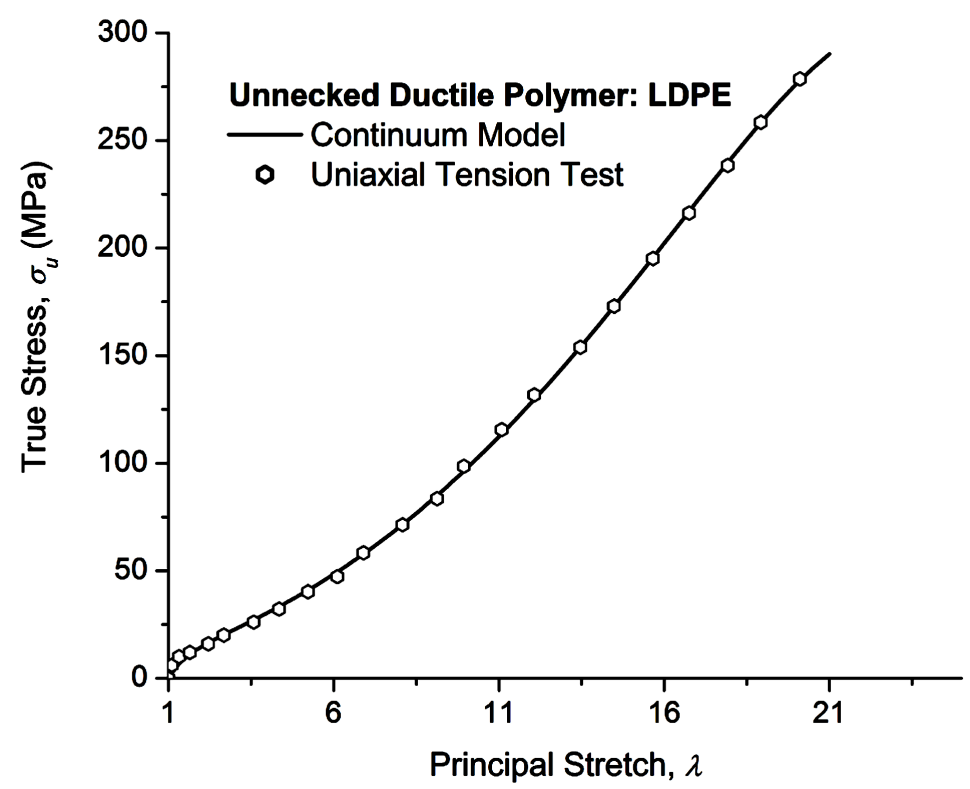

Figure 3. Continuum modeling for ductile polymer material LDPE.

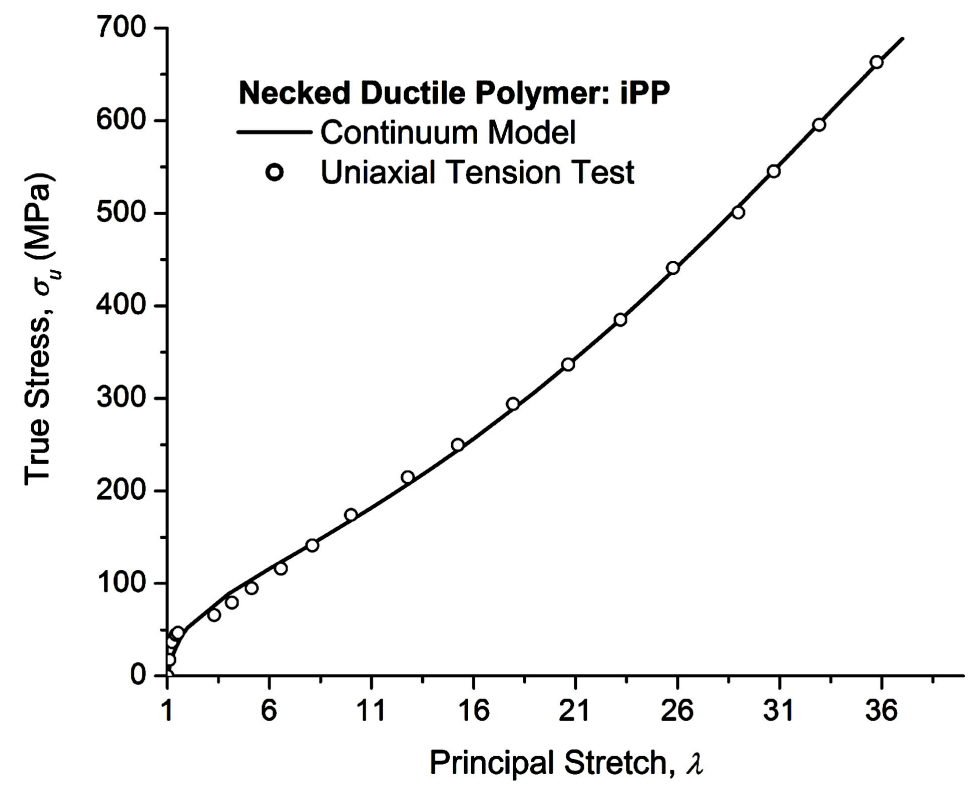

Figure 4. Continuum modeling for necked ductile polymer material iPP.

\subsection{Modeling for Elastomeric Polymeric Materials}

Rubber exhibits finite elastic deformations under tensile loading and fully recovered deformations after unloading.

The benchmark experimental data of vulcanized rubber containing $8 \%$ sulfur was accomplished by Treloar (1944) [16]. The continuum constitutive model is remarkably well suited to fit elastomeric polymers such as the vulcanized rubber with $8 \%$ sulfur. The three coefficients of $c_{1}=0.1409441 \mathrm{MPa}$, $c_{2}=0.1425925 \mathrm{MPa}$, and $c_{3}=3.19703 \times 10^{-7} \mathrm{MPa}$ and detailed plots have been obtained by Zhao (2016) [11]. 


\section{Discussion}

\subsection{Physical Interpretations}

The general solution (10) of the CSE partial differential Equation (8) establishes the generic CSE function for isotropic materials under isothermal processes. Based on the requirements of translational, rotational, and ellipsoidal deformations, the general solution boils down to a particular three-component solution, in which mechanical behavior of materials is concisely described by the three constants: $c_{1}, c_{2}$, and $c_{3}$. In the three-component CSE function (13) for isotropic materials, the first component, $c_{1}\left(I_{1}-3\right)$, represents the work done of normal stress and translational deformation. The second component, $c_{2}\left(\sqrt{I_{2}}-\sqrt{3}\right)$, describes the work done of shear stress and rotational deformation. The third component, $c_{3}\left(I_{1}^{4} / I_{3}-81\right)$, captures the work done of volumetric stress and ellipsoidal deformation.

Physical relevancies of the CSE function are further demonstrated through uniaxial tension tests with the fitted constants for selected polymeric materials with different deformation mechanisms. In addition to the representative materials, the curve-fitting results of PMMA (PLEXIGLAS), HDPE by Nitta and Yamana (2012) [15], and Entec Enflex S4035A thermoplastic elastomer (TPE) by Zhao (2016) [11] are also included. The specific values of the three constants for the seven materials with the three deformation mechanisms are listed in Table 1.

\subsection{Damage Modes for Different Deformation Mechanisms}

\subsubsection{Brittle Polymer: GPPS}

For the GPPS row in Table 1, the largest positive value is $c_{1}$, indicating that the dominant deformation is a translational deformation since the first term of the continuum constitutive model represents translational deformations produced by normal stresses.

Damage modes for polymers ${ }^{2}$. have been revealed through the CSE function. The CSE function predicts that the fractures of brittle material GPPS are due to both shearing and ellipsoidal volumetric damaging. The second term in the continuum constitutive model corresponds to a shear stress and a rotational

Table 1. Constitutive constants with different deformation mechanisms.

\begin{tabular}{ccccc}
\hline Material & $\mathcal{c}_{1}(\mathrm{MPa})$ & $\mathcal{c}_{2}(\mathrm{MPa})$ & $\mathcal{c}_{3}(\mathrm{MPa})$ & Mechanism \\
\hline GPPS & 362341.55 & -41595.99 & -3237.57 & Brittle \\
PMMA & 66039.41 & -17555.19 & -560.67 & Brittle \\
LDPE & 0.3235954 & 14.6382743 & $-1.40847 \times 10^{-10}$ & Ductile \\
iPP & 0.1680651 & 60.2789629 & $-1.09346 \times 10^{-12}$ & Ductile, Necked \\
HDPE & 0.1508911 & 50.2522535 & $-1.68669 \times 10^{-13}$ & Ductile, Necked \\
Rubber & 0.1409441 & 0.1425925 & $3.19703 \times 10^{-7}$ & Elastomeric \\
S4035A & 0.1027292 & 0.0310506 & $2.03450 \times 10^{-7}$ & Elastomeric \\
\hline
\end{tabular}

${ }^{2}$ A polymer fails by either shearing or crazing, as pointed out by Kausch (1987) [17]. 
deformation. Thus, the negative second term describes the damage in shearing mode. The third term in the continuum constitutive model corresponds to a volumetric stress and an ellipsoidal deformation. Thus, the negative third term captures the damage in the ellipsoidal volumetric opening mode. This mode will be further described in ductile polymer cases.

\subsubsection{Ductile Polymers: LDPE and iPP}

For the LDPE and iPP rows in Table 1 , the positive values of $c_{1}$ and $c_{2}$ indicate that the dominant deformations are translational and rotational deformations. The negative values of $c_{3}$ for both LDPE and iPP indicate that elastic-plastic deformations of ductile polymers experience ellipsoidal volumetric damages. Ellipsoidal volumetric damage accelerates at relatively large stretches. Ductile fractures can be described in three stages. ${ }^{3}$ The scanning electron microscopy (SEM) micrographies show large ellipsoidal cavities for isotactic polypropylene filled with ethylene-propylene rubber particles (iPP/EPR) produced by the tensile tests by Mae, Omiya, and Kishimoto (2008) [19] and by Ponçot, Addiego, and Dahoun (2013) [9].

\subsubsection{Elastomeric Polymer: Vulcanized Rubber with $8 \%$ Sulfur}

The positive values of $c_{1}, C_{2}$, and $c_{3}$ for rubber under uniaxial tension tests predict no damage in the combination of translational, rotational, and ellipsoidal volumetric deformations within the test range.

The $c_{1} I_{1}$ term was used in modeling elastomeric polymers. Stored energy functions with only $I_{1}$ terms, however, will fail to model simple torsion experiments for isotropic elastomeric polymers demonstrated by Horgan and Saccomandi (1999) [20]. The $c_{2} \sqrt{I_{2}}$ term accurately describe shear stresses and rotational deformations for isotropic materials.

The $c_{3} I_{1}^{4} / I_{3}$ term captures the ellipsoidal volumetric strengthening for elastomeric polymers at finitely large stretches.

\subsection{Equivalent Stresses and Elastic-Plastic Deformations}

The key to the yield criterion, hardening law, and flow rule formulations is the von Mises equivalent stress. Thus, the von Mises equivalent stress and strain play a crucial role in existing theories of plasticity documented by Khan and Huang (1995) [21]. The von Mises equivalent stress and strain are given respectively by

$$
\overline{\mathrm{S}}=\sqrt{\frac{3}{2} \boldsymbol{S}^{\prime}: \boldsymbol{S}^{\prime}}=\sqrt{\frac{3}{2} \boldsymbol{S}: \boldsymbol{S}-\frac{1}{2}(\operatorname{tr} \boldsymbol{S})^{2}}
$$

and

$$
\overline{\mathrm{E}}=\frac{\sqrt{2}}{3} \sqrt{\left[\ln \left(\frac{\lambda_{1}}{\lambda_{2}}\right)\right]^{2}+\left[\ln \left(\frac{\lambda_{2}}{\lambda_{3}}\right)\right]^{2}+\left[\ln \left(\frac{\lambda_{3}}{\lambda_{1}}\right)\right]^{2}}
$$

where $S^{\prime}$ is the deviatoric stress tensor, $\overline{\mathrm{S}}$ is the von Mises equivalent stress,

${ }^{3}$ The three stages of ductile fractures are void nucleation, void growth, and void coalescence, as summarized by Garrison and Moody (1987) [18]. 
and $\overline{\mathrm{E}}$ is the equivalent logarithmic strain in terms of principal stretches.

Stress-based formulations, however, possess some significant shortcomings. Substituting (2) into (19) $)_{2}$, applying the Cayley-Hamilton equation, collecting terms, and simplifying yields the von Mises equivalent stress in terms of the CSE function

$$
\bar{S}=2 \sqrt{\left(I_{1}^{2}-3 I_{2}\right)\left(\frac{\partial \Psi}{\partial I_{2}}\right)^{2}+\left(I_{2}^{2}-3 I_{1} I_{3}\right)\left(\frac{\partial \Psi}{\partial I_{3}}\right)^{2}+\left(I_{1} I_{2}-9 I_{3}\right) \frac{\partial \Psi}{\partial I_{2}} \frac{\partial \Psi}{\partial I_{3}}}
$$

where no $\partial \Psi / \partial I_{1}$ related terms are noticed.

The Kirchhoff stress tensor, $\tau$, can be converted from the second Piola-Kirchhoff stress tensor by the push-forward operation by $\boldsymbol{F}$

$$
\tau=\sqrt{I_{3}} \sigma=F_{S F}{ }^{\mathrm{T}}
$$

where $\sigma$ is the Cauchy stress tensor or the true stress tensor.

With the CSE function and $I_{3}=1$, the von Mises equivalent stress (21) in terms of invariants boils down to

$$
\bar{S}_{p}=c_{2} \sqrt{I_{1}^{2} / I_{2}-3}
$$

The $c_{2}$ term in (23) indicates that shear stresses drive elastic-plastic deformations. Clearly, the contribution of the $c_{1}$ and $c_{3}$ terms in the CSE function is excluded from the von Mises equivalent stress equation since it was formulated on the assumption that plastic deformations depend only on shear stress instead of normal stress and volumetric stress. Many studies in the past and present show that the von Mises equivalent stress does not produce very good results for isotropic as well as anisotropic ductile metallic materials by Michno and Findley (1976) [10] and by Barsanescu and Comanici (2017) [22], which have far less deformation ranges than those of ductile polymeric materials.

In order to better understand finite elastic-plastic deformations of polymers, $c_{1}, c_{2}$, and $c_{3}$ terms in the continuum constitutive model are individually depicted for both LDPE and iPP ductile polymers. The true stress-stretch component curves for both LDPE and iPP are shown in Figure 5 and Figure 6, respectively.

All three terms of the continuum constitutive model contribute to elasticplastic deformations. ${ }^{4}$ The first term and the second term are the major contributors to elastic-plastic deformations, meaning that both the normal stress and the shear stress contribute to elastic-plastic deformations. As the stretch increases, both normal stress and shear stress increase. Between the curves of the first term and the second term, there exists a break-even stretch, $\lambda_{b}$, defined in (18). When principal stretches are much smaller than the break-even stretch, the rotational deformation dominates elastic-plastic deformations and shear stress contributes more than normal stress. At the break-even stretch, shear stress

${ }^{4}$ Deformations of ductile polymers are the chain orientation and the volume damage, mentioned by Ponçot, Addiego, and Dahoun (2013) [9]. 


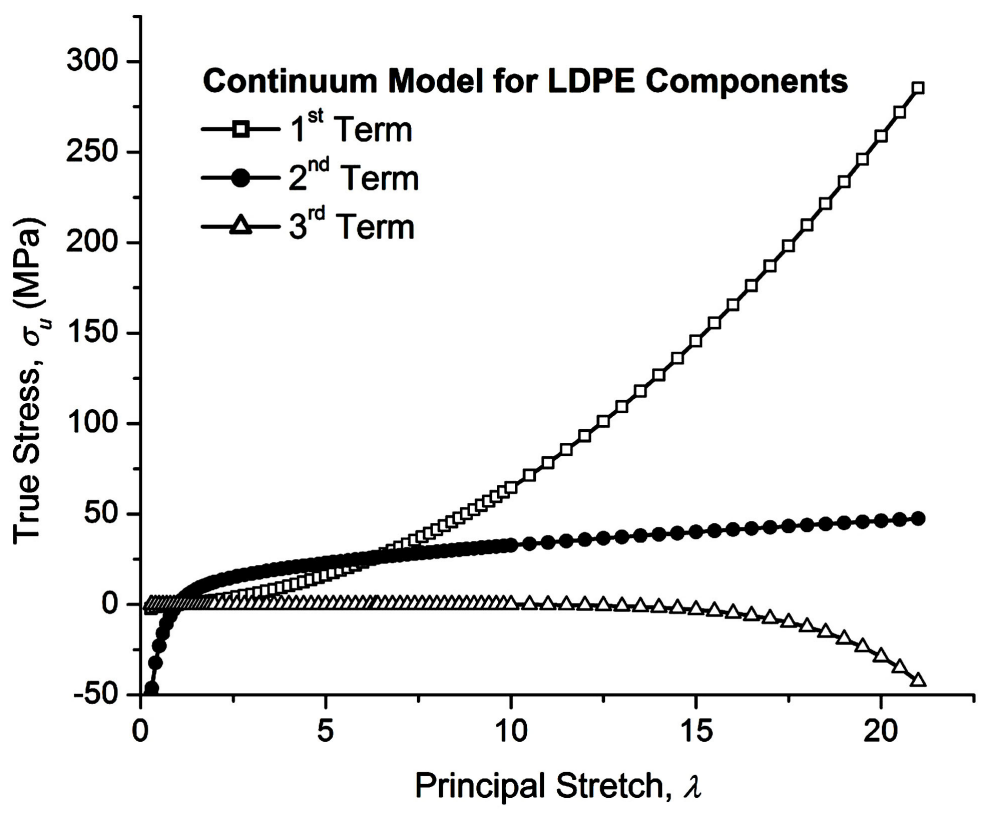

Figure 5. True stress-stretch component curves for ductile polymer LDPE.

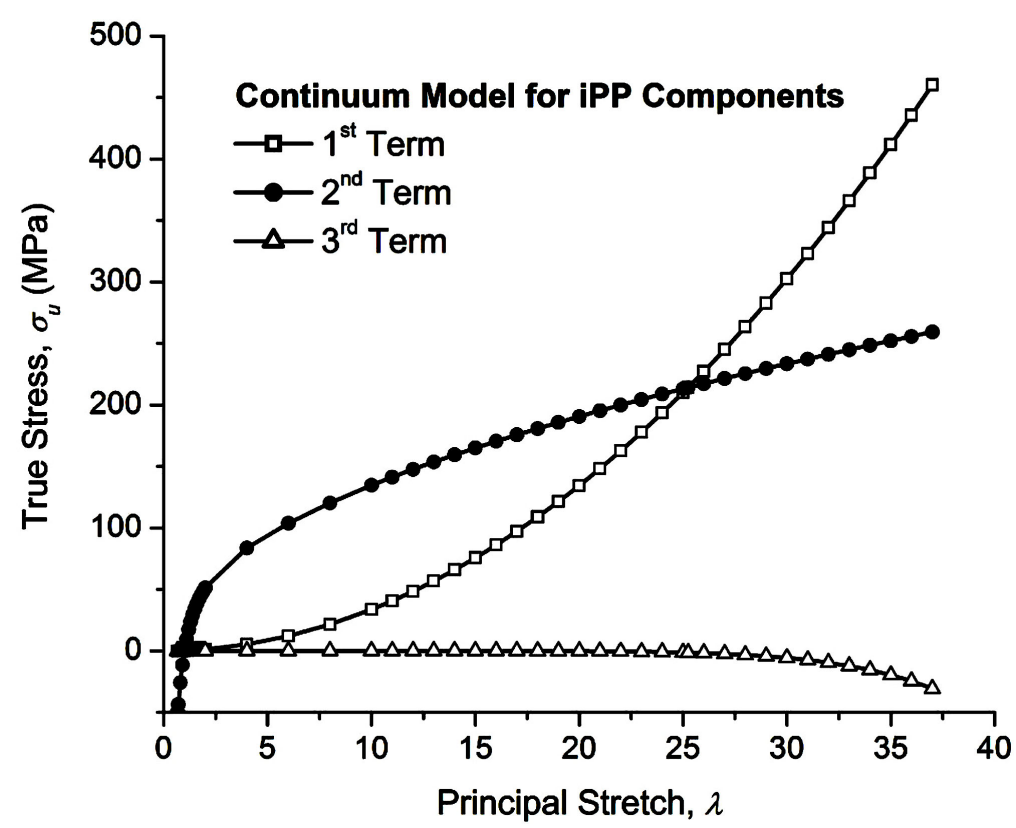

Figure 6. True stress-stretch component curves for necked ductile polymer iPP.

contributes the same as normal stress. When principal stretches are much greater than the break-even stretch, the translational deformation dominates further elastic-plastic deformations and normal stress contributes more than shear stress. The third term only contributes minor volumetric damages at very large stretches. The break-even stretches for the selected ductile polymers have been evaluated and determined as $\lambda_{b}=6.34374$ for LDPE and $\lambda_{b}=25.24009$ for iPP, respectively. As stretches fall within 0 and 1 , deformations are compressive. In compressive deformations, shear stress indeed dominates plastic 
deformations while normal stress contributes little as predicted and depicted in both Figure 5 and Figure 6. In compressive deformations, shear stress dominates but normal stress cannot be ignored at very small stretches although no compressive break-even stretches exist. Thus, the shear stress dominated equivalent stress is only approximately fit for both small tensile and compressive elastic-plastic deformations.

The equivalently converted stress-strain curves in different deformation modes should coincide with its uniaxial tension stress-strain curve for both elastic and elastic-plastic deformations. The equivalent stress (21), along with (22), and the equivalent logarithmic strain (20) for Treloar's experimental data in uniaxial tension, equibiaxial tension, and pure shear modes have been calculated and depicted in Figure 7. Similarly, the equivalent true stress and equivalent true strain curves for iPP have been calculated from its uniaxial tension data, the continuum model predicted equibiaxial tension and pure shear data and plotted in Figure 8. In both cases, the von Mises stresses at different deformation modes are only equivalent at very small strains.

Unlike the von Mises equivalent stress or the equivalent strain, the CSE function captures the finite elastic-plastic deformations of ductile polymers in the following aspects:

1) Different combinations of three coefficients for the selected ductile polymers given in Table 1 indicate that the contributions of normal stress, shear stress, and volumetric stress on elastic-plastic deformations vary with materials.

2) There exist tensile break-even stretches for finite deformations of the selected ductile polymers defined in (18) and shown in Figure 5 and Figure 6, demonstrating that the proportion of a normal stress and a shear stress of the same material changes with principal stretches.

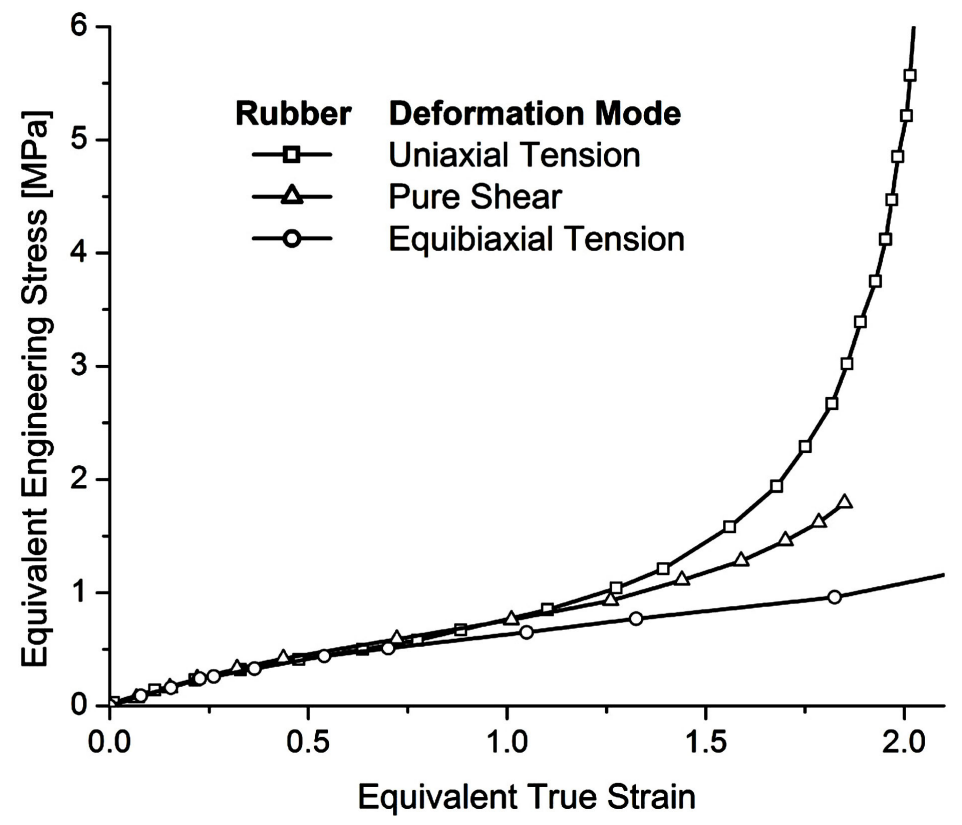

Figure 7. Equivalent engineering stress-equivalent true strain curves for rubber. 


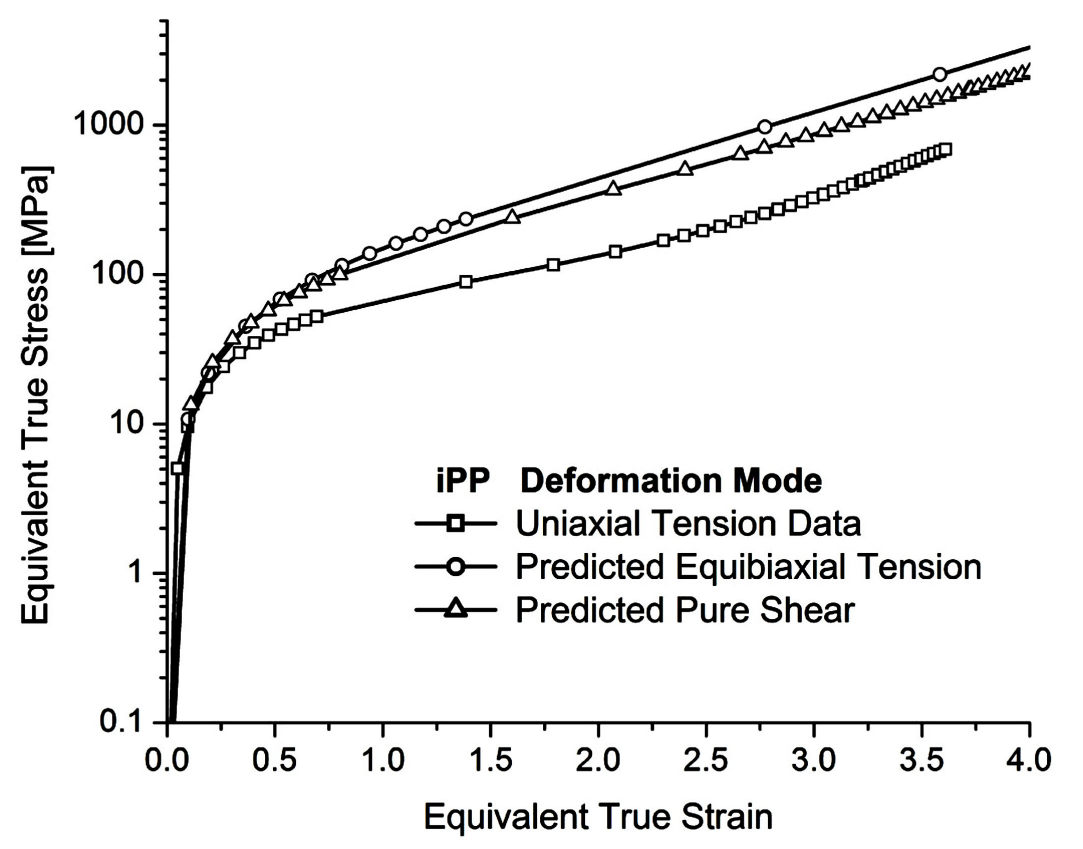

Figure 8. Equivalent true stress-equivalent true strain curves for iPP.

3) The values of $c_{3}$ in Table 1 for the selected ductile polymers are all negative, implying energy dissipation and capturing corresponding volumetric damage.

4) The combination of three invariants of stretches in the CSE function (11) possesses three different deformation components, generating physically relevant deformations.

5) The CSE function is a physically relevant and mathematically covariant model established from continuum mechanics, predicting deformations in other untested modes.

Thus, a new algorithm based on the CSE function for the finite elasticityplasticity theory is recommended for future research and development.

\subsection{Common Practices in Constitutive Modeling of Polymers}

The most commonly used experimental tests for material characterizations are uniaxial tension, pure shear, equibiaxial tension, uniaxial compressions, confined compression, and torsion tests, as reviewed by Charlton, Yang, and Teh (1994) [23].

Common practices to characterize and model elastomeric polymers are to take the uniaxial tension, pure shear, and equibiaxial tension tests, to fit several theoretical models with the three test results, and to select the best possible theoretical model based on accuracy, stability, and efficiency. Among the three tests, the pure shear test can produce the same accuracy as the uniaxial tension test, but the deformation range is far less than that of the uniaxial tension. The equibiaxial tension fails to match both the accuracy and the deformation range of the uniaxial tension. Thus, the uniaxial tension test is the best method 
available to characterize the mechanical behavior of materials. When theoretical constitutive models are fitted by the test in one mode, predictions of other deformation modes will indicate the quality of theoretical models, as noticed by Steinmann, Hossain, and Possart (2012) [24]. When constitutive models are fitted by the three tests, let alone the errors introduced by the tests with different accuracies, material costs, sample preparations, extra tests, and data treatments, the prediction of the deformations other than the three deformation modes are still suspicious if the constitutive models do not possess a full predictability. In other words, fully predictive models and uniaxial tests are the best combination for constitutive modeling of polymeric materials.

To characterize and model finite deformations of ductile polymers, uniaxial tension and compression tests are usually used. Intrinsic material nonlinearities, however, could be coupled with boundary and geometric nonlinearities during experimental tests. In order to capture intrinsic material nonlinearities, symmetries should also be applied in experimental designs and tests. For necked specimen in uniaxial tension tests, their deformations are no longer homogeneous and traditional extensometers are no longer accurate. Video-based extensometers by G'sell and Jonas (1979) [25] and G'sell, Hiver, and Dahoun (2002) [26] and the laser speckle extensometer combined with the digital image correlation method by Laraba-Abbes, Ienny, and Piques (2003) [27] have been applied to deal with inhomogeneous deformations in uniaxial tension tests. In uniaxial compression tests, for standard compression samples as well as tension samples done by Poulain et al. (2013) [28], barreling occurs during large compressions. Thus, compressive strengths are usually overestimated due to inhomogeneous deformations, non-uniform force distributions, and hydrostatic pressure effects. The extra work for barreling is unavoidably counted as part of the total work for uniaxial compressions. Unlike uniaxial tension tests, non-uniform force distributions and hydrostatic pressure effects in uniaxial compression tests become worse as the compression goes. An integrated computational-experimental method has been developed to circumvent the overestimation of compressive elastic-plastic stress-strain curves for ductile polymeric thin films by Zhao (1998) [29]. For predictions of finite elastic-plastic deformations in other modes, however, von-Mises stress-based equations were traditionally used. Since the von-Mises equivalent stress is only approximately accurate within a narrow stretching range, predictions of untested deformation modes for ductile polymeric materials by these types of theories are questionable.

In the CSE constitutive modeling for isotropic polymeric materials, both experimental characterizations and theoretical predictions are indispensible. Experimental tests are used to determine the three unknown constitutive constants in the CSE function for a specific material. The CSE function as a theoretical model is applied to predict the deformations of untested deformation modes since exclusive experimental tests cannot cover all possible deformation 
modes. For isotropic polymeric materials with different deformation mechanisms, applications of the CSE function and uniaxial tension tests minimize errors and tests in traditional constitutive modeling processes.

\section{Conclusions}

The CSE function, originally developed for modeling elastomeric polymers, has been extended in its application to brittle and ductile polymers. The CSE function fits uniaxial tension data for GPPS and PMMA as brittle polymers, LDPE, iPP, and HDPE as ductile polymer materials both with and without necking, and vulcanized rubber containing 8\% sulfur and Entec Enflex S4035A TPE as elastomeric polymers remarkably well.

The CSE function as a physically relevant and mathematically covariant model elucidates the three major deformation mechanisms of polymers. For brittle polymer materials, deformations are mainly elastic, then slightly elastic-plastic deformations before fracture, and the failure modes are both shearing and ellipsoidal volumetric opening. For ductile polymeric materials, deformations begin with slightly elastic, then mainly elastic-plastic, and ellipsoidal volume damages at relatively large stretches. For elastomeric polymeric materials, deformations are rather homogeneous and fully elastic with no damages within the testing range.

The difference between brittle and ductile elastic-plastic deformations is that brittle polymers take little shearing deformation while ductile polymers endure both finite translational deformation by normal stress and rotational deformation by shear stress.

The von Mises equivalent stress has been studied in terms of the CSE function. In large enough tensile deformations, all three terms in the continuum constitutive model contribute to elastic-plastic deformations and a break-even stretch exists between the normal stress component curve and the shear stress component curve. The von Mises equivalent stress is only approximately accurate within a narrow stretching range.

Common practices in constitutive modeling of polymeric materials have been briefly reviewed, the advantages and disadvantages of existing models and testing methods have been concisely discussed, and the CSE function and uniaxial tension tests have been selected for the constitutive modeling of polymeric materials. A new algorithm based on the CSE function for the finite elasticity-plasticity theory of ductile polymers has been evident.

\section{Acknowledgements}

The author is immensely grateful to Ampere A. Tseng and Tei-Chen Chen for their encouragement. He is also very thankful to Youyi Chu for useful discussions in the field of materials science.

He would also like to show his gratitude to Jianming and Jiesi Zhao for their help in many ways. 


\section{References}

[1] Truesdell, C. and Noll, W. (1965) The Non-Linear Field Theories of Mechanics, In: Flügge, S., Ed., Encyclopedia of Physics, Springer-Verlag, Berlin, Vol. 3. https://doi.org/10.1007/978-3-642-46015-9_1

[2] Hill, R. (1950) The Mathematical Theory of Plasticity. Clarendon Press, Oxford.

[3] Simo, J.C. and Hughes, T.J.R. (1998) Computational Inelasticity. Springer-Verlag, New York.

[4] Haupt, P. (2002) Continuum Mechanics and Theory of Materials. 2nd Edition, Springer-Verlag, Berlin. https://doi.org/10.1007/978-3-662-04775-0

[5] Armen, H. (1979) Assumptions, Models, and Computational Methods for Plasticity. Computers \& Structures, 10, 161-174. https://doi.org/10.1016/0045-7949(79)90084-1

[6] Naghdi, P.M. (1990) A Critical Review of the State of Finite Plasticity. Journal of Applied Mathematics and Physics (ZAMP), 41, 315-394. https://doi.org/10.1007/BF00959986

[7] Xiao, H., Bruhns, O.T. and Meyers, A. (2006) Elastoplasticity beyond Small Deformations. Acta Mechanica, 182, 31-111. https://doi.org/10.1007/s00707-005-0282-7

[8] Chaboche, J.L. (2008) A Review of Some Plasticity and Viscoplasticity Constitutive Theories. International Journal of Plasticity, 24, 1642-1693.

https://doi.org/10.1016/j.ijplas.2008.03.009

[9] Ponçot, M., Addiego, F. and Dahoun, A. (2013) True Intrinsic Mechanical Behaviour of Semi-Crystalline and Amorphous Polymers: Influences of Volume Deformation and Cavities Shape. International Journal of Plasticity, 40, 126-139. https://doi.org/10.1016/j.ijplas.2012.07.007

[10] Michno, M.J. and Findley, W.N. (1976) An Historical Perspective of Yield Surface Investigations for Metals. International Journal of Non-Linear Mechanics, 11, 59-82. https://doi.org/10.1016/0020-7462(76)90039-1

[11] Zhao, F.Z. (2016) Continuum Constitutive Modeling for Isotropic Hyperelastic Materials. Advances in Pure Mathematics, 6, 571-582. https://doi.org/10.4236/apm.2016.69046

[12] Holzapfel, G.A. (2000) Nonlinear Solid Mechanics. John Wiley \& Sons, Chichester.

[13] Meyers, M.A. and Chawla, K.K. (2009) Mechanical Behavior of Materials. 2nd Edition, Cambridge University Press, Cambridge.

[14] Smith, W.F. (1993) Foundations of Materials Science and Engineering. McGrow-Hill, Inc., New York.

[15] Nitta, K. and Yamana, M. (2012) Poisson's Ratio and Mechanical Nonlinearity under Tensile Deformation in Crystalline Polymers, In: Vicente, J.D., Ed., Rheology, InTech, Rijeka, 113-132. https://doi.org/10.5772/34881

[16] Treloar, L.R.G. (1944) Stress-Strain Data for Vulcanised Rubber under Various Types of Deformation. Transactions of the Faraday Society, 40, 59-70. https://doi.org/10.1039/tf9444000059

[17] Kausch, H.-H. (1987) Polymer Fracture. 2nd Edition, Springer-Verlag, Berlin.

[18] Garrison, W.M. and Moody, N.R. (1987) Ductile Fracture. Journal of Physics and Chemistry of Solids, 48, 1035-1074. https://doi.org/10.1016/0022-3697(87)90118-1

[19] Mae, H., Omiya, M. and Kishimoto, K. (2008) Microstructural Observation and Simulation of Micro Damage Evolution of Ternary Polypropylene Blend with Ethylene-Propylene-Rubber (EPR) and Talc. Journal of Solid Mechanics and Materials 
Engineering, 2, 1018-1036. https://doi.org/10.1299/jmmp.2.1018

[20] Horgan, C.O. and Saccomandi, G. (1999) Simple Torsion of Isotropic, Hyperelastic, Incompressible Materials with Limiting Chain Extensibility. Journal of Elasticity, 56, 159 -170. https://doi.org/10.1023/A:1007606909163

[21] Khan, A.S. and Huang, S. (1995) Continuum Theory of Plasticity. John Wiley \& Sons, New York.

[22] Barsanescu, P.D. and Comanici, A.M. (2017) von Mises Hypothesis Revised. Acta Mechanica, 228, 433-446. https://doi.org/10.1007/s00707-016-1706-2

[23] Charlton, D.J., Yang, J. and Teh, K.K. (1994) A Review of Methods to Characterize Rubber Elastic Behavior for Use in Finite Element Analysis. Rubber Chemistry and Technology, 67, 481-503. https://doi.org/10.5254/1.3538686

[24] Steinmann, P., Hossain, M. and Possart, G. (2012) Hyperelastic Models for Rubber-Like Materials: Consistent Tangent Operators and Suitability for Treloar Data. Archive of Applied Mechanics, 82, 1183-1217. https://doi.org/10.1007/s00419-012-0610-z

[25] G'sell, C. and Jonas, J.J. (1979) Determination of the Plastic Behavior of Solid Polymers at Constant True Strain Rate. Journal of Materials Science, 14, 583-591. https://doi.org/10.1007/BF00772717

[26] G'sell, C., Hiver, J.M. and Dahoun, A. (2002) Experimental Characterization of Deformation Damage in Solid Polymers under Tension, and Its Interrelation with Necking. International Journal of Solids and Structures, 39, 3857-3872. https://doi.org/10.1016/S0020-7683(02)00184-1

[27] Laraba-Abbes, F., Ienny, P. and Piques, R. (2003) A New Tailor-Made Methodology for the Mechanical Behavior Analysis of Rubber-Like Materials: I. Kinematics Measurements Using a Digital Speckle Extensometry. Polymer, 44, 807-820. https://doi.org/10.1016/S0032-3861(02)00718-8

[28] Poulain, X., Kohlman, L.W., Binienda, W., Roberts, G.D., Goldberg, R.K. and Benzerga, A.A. (2013) Determination of the Intrinsic Behavior of Polymers Using Digital Image Correlation Combined with Video-Monitored Testing. International Journal of Solids and Structures, 50, 1869-1878. https://doi.org/10.1016/j.ijsolstr.2013.01.041

[29] Zhao, F. (1998) Modeling for Mechanical Behavior of Thin Films. Ph.D. Dissertation, Drexel University, Philadelphia. 